\title{
Hemangiomas infantiles
}

\section{Infantile hemangiomas.}

Hernández-Zepeda $\mathrm{C}^{1}$, García-Romero $\mathrm{MT}^{2}$

\section{INTRODUCCIÓN}

Las lesiones vasculares en lactantes y niños se clasifican principalmente en dos grupos: tumores y malformaciones vasculares. ${ }^{1}$ Los hemangiomas infantiles se encuentran dentro del grupo de tumores benignos junto con otros como el granuloma piógeno, hemangioendotelioma kaposiforme y el hemangioma en penacho. Sin embargo, los hemangiomas infantiles son los tumores vasculares más comunes. ${ }^{2}$

Los hemangiomas infantiles se caracterizan por tener una fase de crecimiento, de estabilidad y de involución; en contraste con las malformaciones vasculares que están conformadas por capilares, arterias, venas y vasos linfáticos anómalos y crecen de manera proporcional al crecimiento del niño, generalmente sin involucionar. ${ }^{2}$ Existen otros hemangiomas poco comunes con una rápida involución ( $\mathrm{RICH}$ ) o hemangiomas que no involucionan $(\mathrm{NICH})$; distintos a los hemangiomas infantiles. ${ }^{1}$

\section{EPIDEMIOLOGÍA}

La verdadera incidencia de los hemangiomas infantiles se desconoce, pero se estima que cerca del $10 \%$ de los niños caucásicos los presentan. ${ }^{3}$ Algunos factores que predisponen a su aparición son: género femenino (de 2-3 veces más riesgo); ${ }^{4}$ ser pre-término y con bajo peso al nacer; ${ }^{5}$ productos de embarazos múltiples; edad materna avanzada; placenta previa o alguna otra anomalía placentaria. $^{5}$

\section{CUADRO CLÍNICO}

La mayoría de los hemangiomas infantiles no son clínicamente evidentes al nacimiento, sino en los primeros días a semanas de
${ }^{1}$ Médico pasante del servicio social en investigación dermatología pediátrica.

${ }^{2}$ Médico adscrito al departamento de dermatología.

Instituto Nacional de Pediatría, México.

Recibido: 5 de enero del 2017

Aceptado: 7 de febrero del 2017

Correspondencia

Dra. María Teresa García Romero

teregarro@gmail.com

Este artículo debe citarse como

Hernández-Zepeda C, García-Romero MT. Hemangiomas infantiles. Acta Pediatr Mex. 2017;38(3):202-207. 
vida. ${ }^{6}$ En muchos casos se encuentra una lesión premonitoria que es una placa violácea o con telangiectasias y una zona de vasoconstricción periférica. ${ }^{2}$ Generalmente, las lesiones son únicas, aunque en el $20 \%$ de los casos pueden existir múltiples $^{6}$ (Figura 1). Pueden aparecer en cualquier parte de la piel, mucosas, incluso órganos internos como intestino o hígado, pero generalmente lo hacen en cabeza y cuello. ${ }^{2}$ El aspecto clínico depende de la localización y profundidad; de acuerdo a su profundidad se clasifican en:

- Superficiales (más comunes): pápula o nódulo de color rojizo sobre piel clínicamente normal. ${ }^{7}$ Sus sinónimos son hemangiomas capilares o en fresa, pero el término correcto es hemangioma infantil superficial. $^{8}$

- Profundos: nódulos subcutáneos de tonalidad azulada con telangiectasias centrales, ${ }^{2}$ su sinónimo es hemangioma cavernoso, pero este término es confuso y es mejor evitarlo. $^{2}$

- Mixtos: con características clínicas tanto superficiales como profundas $^{7}$ (Figura 2).

De acuerdo a su localización pueden clasificarse como:

- Localizados: los más comunes, generalmente en cara cerca de la línea media.

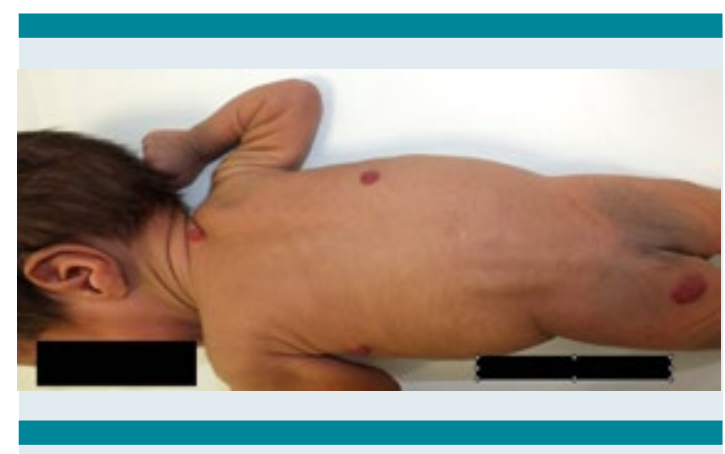

Figura 1. Hemangiomatosis múltiple.

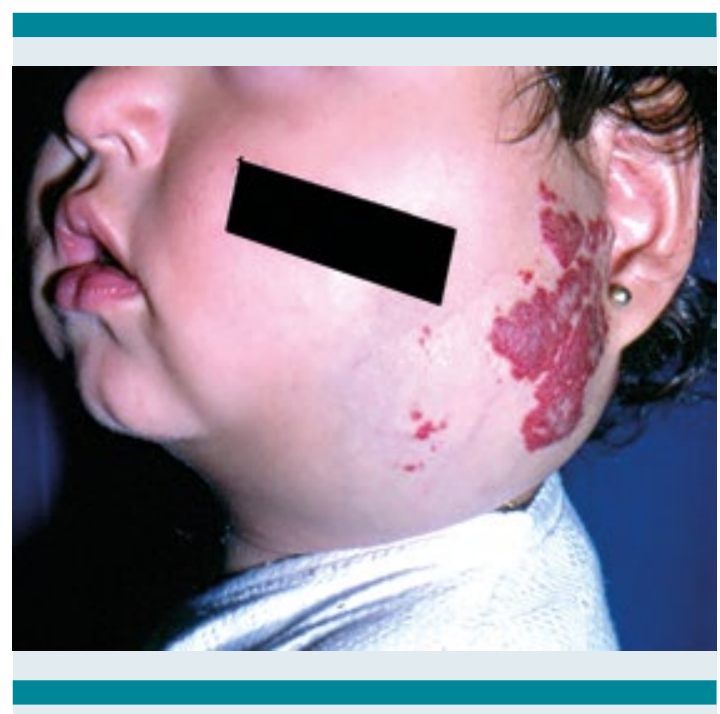

Figura 2. Hemangioma mixto: componentes superficial y profundo.

- Segmentarios: generalmente afectan un territorio cutáneo específico sin pasar línea media $^{9}$ (Figura 3) y requieren un tratamiento más intensivo y prolongado en comparación con los hemangiomas localizados. ${ }^{2}$ Además, pueden asociarse a síndromes como PHACES y LUMBAR.

Los hemangiomas infantiles tienen tres fases:

1. Fase de proliferación que consta de una etapa de crecimiento rápido durante los primeros 5 meses de vida, en la cual crece el $80 \%$ de su tamaño final, y a partir del $6^{\circ}$ a $12^{\text {vo }}$ mes ocurre la fase de crecimiento lenta. ${ }^{1,3}$ La proliferación después del año de vida puede ocurrir, pero es poco común. ${ }^{3}$

2. Fase de estabilidad o meseta en la que el hemangioma deja de proliferar, puede sobreponerse con la fase de crecimiento lenta.

3. Fase de involución espontánea que típicamente inicia a partir del año de edad y continúa a lo largo de los años, se estima que el tamaño del hemangioma disminuye 


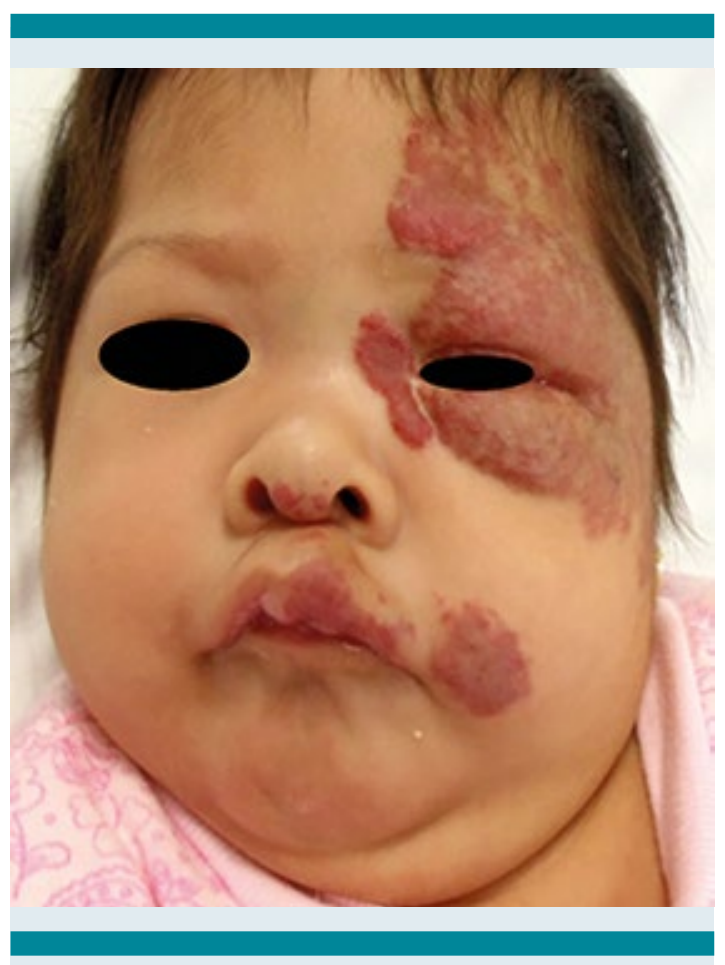

Figura 3. Hemangioma segmentario.

$10 \%$ al año, por lo que a los 5 años habrá involucionado el $50 \%$ aproximadamente.

Los hemangiomas infantiles pueden ser marcadores de síndromes con distintas malformaciones según la topografía, como:

A. Síndrome de PHACE: acrónimo en inglés para describir un síndrome neurovascular, el cual está definido por las características enlistadas en el (Cuadro 1) (9-12 $^{9}$ diagnóstico de PHACE es clínico por la presencia de un hemangioma segmentario más una o más de las anomalías previamente descritas. Los pacientes en los que se sospeche este síndrome deberán ser evaluados por oftalmología, neurología, cardiología y dermatología. Se sugiere realizar angiorresonancia magnética nuclear de cabeza, cuello y pecho, para descartar o confirmar anomalías. ${ }^{2,10}$
Cuadro 1. Síndrome de PHACE

\begin{tabular}{|c|c|c|}
\hline & Anomalía & Descripción \\
\hline$P$ & $\begin{array}{l}\text { Anomalías de fosa } \\
\text { posterior } \\
\text { (anomalía extracutánea } \\
\text { más común })^{9}\end{array}$ & $\begin{array}{l}\text { Más común es el com- } \\
\text { plejo Dandy-Walker que } \\
\text { consta de hipoplasia } \\
\text { cerebral o atrofia o } \\
\text { disgenesia/agenesia del } \text { vermis }^{10}\end{array}$ \\
\hline $\mathrm{H}$ & Hemangioma & $\begin{array}{l}\text { Grande, segmentario, } \\
\text { facial o cefálico, afecta } \\
\text { de uno a varios } \\
\text { dermatomas }\end{array}$ \\
\hline A & Anomalías arteriales & $\begin{array}{l}\text { Persistencia de arterias } \\
\text { embrionarias y } \\
\text { agenesia de la arteria } \\
\text { carótida o vertebral }\end{array}$ \\
\hline $\mathrm{C}$ & $\begin{array}{l}\text { Cardiacas (segunda } \\
\text { manifestación } \\
\text { extracutánea más } \\
\text { común) }\end{array}$ & $\begin{array}{l}\text { Coartación de la aorta, } \\
\text { anormalidades del arco } \\
\text { aórtico, defectos } \\
\text { ventriculares o septales }{ }^{11}\end{array}$ \\
\hline$E$ & Oculares & $\begin{array}{l}\text { Microoftalmos, } \\
\text { persistencia de venas } \\
\text { fetales y atrofia del } \\
\text { nervio óptico }\end{array}$ \\
\hline
\end{tabular}

B. Síndrome LUMBAR, acrónimo de las características enlistadas en el Cuadro 2. Es necesario que estos pacientes sean evaluados por urología, neurología y dermatología. ${ }^{13-16}$

\section{DIAGNÓSTICO}

El diagnóstico de los hemangiomas infantiles es clínico; sin embargo, en los casos en los hubiera duda, se puede realizar una biopsia de piel, en la que se observarían vasos capilares prolifera-

Cuadro 2. Síndrome LUMBAR

\begin{tabular}{|ll} 
L & Lower-body hemangioma \\
U & Ulceración \\
M & Mielopatía \\
\hline B & Anormalidades óseas \\
\hline A & Malformaciones anorrectales \\
R & Malformaciones renales \\
\hline
\end{tabular}


tivos con marcador GLUT1 positivo, lo cual es patognomónico. ${ }^{17}$

\section{DIAGNÓSTICOS DIFERENCIALES}

Los hemangiomas infantiles segmentarios en cara pueden confundirse tempranamente con una malformación capilar o mancha en vino de oporto segmentaria (asociada o no a síndrome de Sturge-Weber). ${ }^{11}$ Una diferencia importante es que la malformación capilar no va a proliferar ni involucionar. ${ }^{18}$

También pueden confundirse con un granuloma piógeno; sin embargo, la edad de aparición y el curso clínico de éstos son muy distintos. Por último, pueden confundirse con los otros tumores vasculares poco comunes como hemangioendotelioma kaposiforme, angioma en penacho y los $\mathrm{RICH}$ o $\mathrm{NICH} .{ }^{19}$

\section{COMPLICACIONES}

La complicación más común es la ulceración, debe sospecharse hemangioma infantil en fase de proliferación rápida, con cambio de coloración antes de los tres meses, y si están localizados en puntos de fricción y maceración. ${ }^{20}$ Puede haber infección o sangrado como consecuencia de la ulceración.

Los hemangiomas infantiles de localización cervicofacial, mandibular o distribución en forma de barba tienen riesgo de obstrucción de vía aérea, que debe sospecharse en pacientes que desarrollan estridor o disfonía progresiva, tos o cianosis. ${ }^{1}$

Los hemangiomas infantiles periorbitarios confieren riesgo de obstrucción del campo visual durante su fase de proliferación y de astigmatismo por la presión ejercida en la córnea. ${ }^{21}$ La evaluación por oftalmología es prioritaria ya que podría haber un daño permanente, incluso ceguera. ${ }^{1}$
Aunque sumamente raro, puede aparecer el fenómeno de Kasabach-Merrit, el cual genera trombocitopenia o coagulopatía severa como resultado del atrapamiento de plaquetas por el hemangioma infantil, aunque generalmente se asocia a hemangioendotelioma kaposiforme y angioma en penacho.

\section{TRATAMIENTO SUGERIDO}

1. Debe ser individualizado según el tamaño, número y localización de las lesiones, para así poder predecir las posibles complicaciones.

2. Consultar/referir al paciente con un dermatólogo pediatra lo más pronto posible, preferentemente antes de o durante la fase de proliferación del hemangioma (primeras semanas de vida).

3. El paciente debe ser valorado constantemente durante la fase proliferativa por el dermatólogo pediatra. Una manera efectiva de llevar un control sobre la lesión es documentándola por medio de fotografías.

4. Educar a la familia sobre el curso natural que tendrá el hemangioma infantil, posibles complicaciones y tratamiento cuando esté indicado, así como sus riesgos y beneficios.

5. Enfatizar que al involucionar el hemangioma infantil la piel puede presentar atrofia, cicatrices, piel redundante o telangiectacias; eventualmente puede requerirse manejo quirúrgico. ${ }^{22,23}$

\section{INDICACIONES PARA INICIAR TRATA- MIENTO}

1. Lesiones que puedan alterar la función adecuada, como son las periorbitarias, aquellas que puedan obstruir vía aérea 


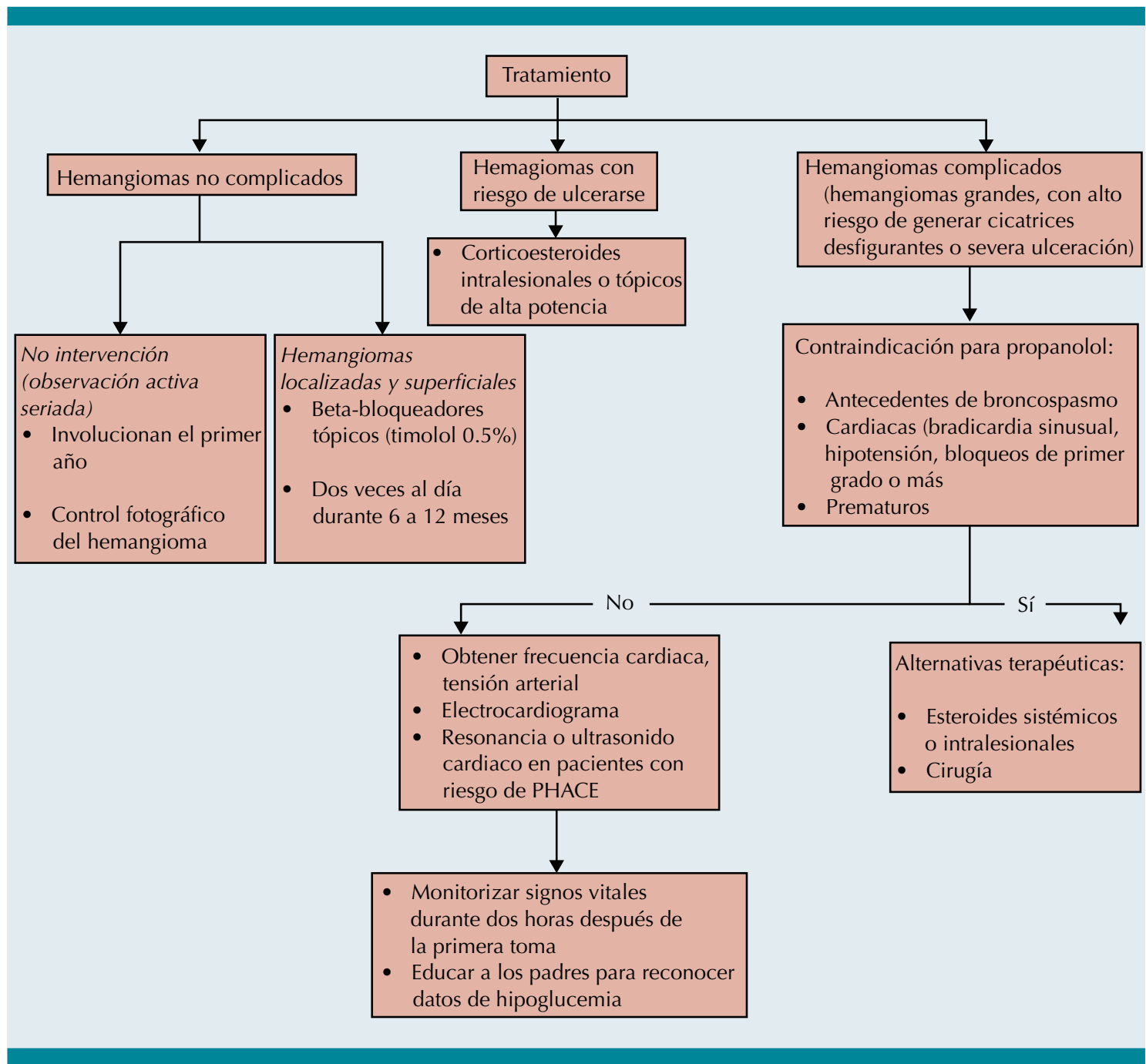

Figura 4. Tratamiento.

o la alimentación, aquellas que generen sangrado como gastrointestinales o alteraciones circulatorias como las hepáticas grandes.

2. Lesiones con alto riesgo de cicatrización o desfiguramiento como segmentarias, periorbitarias, en nariz (nariz de Cyrano), boca y periauriculares; que reciben trauma constante ${ }^{24}$ o que tienen gran tamaño o crecimiento acelerado (Figura 4). ${ }^{25-28}$

\section{REFERENCIAS}

1. Enjolras O, Mulliken JB. Vascular tumors and vascular malformations (new issues). Adv Dermatol. 1997;13:375-423.

2. Denise W Metry. Infantile hemangiomas: Epidemiology, pathogenesis, clinical features, and complications. Upto Date 2016 http://www.uptodate.com/contents/infantilehemangiomas-epidemiology-pathogenesis-clinical-features-and-complications

3. Haggstrom AN, Drolet BA, Baselga E, et al. Prospective study of infantile hemangiomas: clinical characteristics predicting complications and treatment. Pediatrics. 2006;118:882-887. 
4. Hemangioma Investigator Group, Haggstrom AN, Drolet $B A$, et al. Prospective study of infantile hemangiomas: demographic, prenatal, and perinatal characteristics. J Pediatr. 2007;150:291-294.

5. Drolet BA, Esterly NB, Frieden IJ. Hemangiomas in children. N Engl J Med.1999;341:173-181.

6. Chiller KG, Passaro D, Frieden IJ. Hemangiomas of infancy: clinical characteristics, morphologic subtypes, and their relationship to race, ethnicity, and sex. Arch Dermatol. 2002;138:1567-1576.

7. Meter DW, Hebert AA. Benign cutaneous vascular tumors of infancy: when to worry, what to do. Arch Dermatol. 2000;136:905-914.

8. FriedenIN, Eichenfield LF, Esterly NB, et al. Guidelines of care for hemangiomas of infancy. American Academy of Dermatology Guidelines/Outcomes Committee. J Am Acad Dermatol. 1997;37:631-637.

9. Waner M, North PE, Scherer KA, et al. The nonrandom distribution of facial hemangiomas. Arch Dermatol. 2003;139:869-875.

10. Haggstrom AN, Garzon MC, Baselga E, et al. Risk for PHACE syndrome in infants with large facial hemangiomas. Pediatrics. 2010;126:418-426.

11. Poindexter G, Metry DW, Barkovich AJ, Frieden IJ. PHACE syndrome with intracerebral hemangiomas, heterotopia, and endocrine dysfunction. Pediatr Neurol. 2007;36:402-406.

12. Metry D, Heyer G, Hess C, et al. Consensus Statement on Diagnostic Criteria for PHACE Syndrome. Pediatrics. 2009;124:1447-1450.

13. Tubbs RS, Wellons JC 3rd, Iskandar BJ, Oakes WJ. Isolated flat capillary midline lumbosacral hemangiomas as indicators of occult spinal dysraphism. J Neurosurg. 2004;100:86-89.

14. lacobas I, Burrows PE, Frieden IJ, et al. LUMBAR: association between cutaneous infantile hemangiomas of the lower body and regional congenital anomalies. J Pediatr. 2010;157:795-801.

15. Razon MJ, Kräling BM, Mulliken JB, Bischoff J. Increased apoptosis coincides with onset of involution in infantile hemangioma. Microcirculation. 1998;5:189-195.
16. Metry DW, Hawrot A, Altman C, Frieden IJ. Association of solitary, segmental hemangiomas of the skin with visceral hemangiomatosis. Arch Dermatol. 2004;140:591-596.

17. North PE, Waner M, Mizeracki A, Mihm MC Jr. GLUT1: a newly discovered immunohistochemical marker for juvenile hemangiomas. Hum Pathol 2000; 31:11.

18. Martinez-Perez D, Fein NA, Boon LM, Mulliken JB. Not all hemangiomas look like strawberries: uncommon presentations of the most common tumor of infancy. Pediatr Dermatol. 1995;12:1-6.

19. Enjolras O, Mulliken JB. The current management of vascular birthmarks. Pediatr Dermatol. 1993;10:311-313.

20. Kim HJ, Colombo M, Frieden IJ. Ulcerated hemangiomas: clinical characteristics and response to therapy. J Am Acad Dermatol. 2001;44:962-972.

21. Ceisler EJ, Santos L, Blei F. Periocular hemangiomas: what every physician should know. Pediatr Dermatol. 2004;21:1-9.

22. Bauland CG, Lüning TH, Smith JM, et al. Untreated hemangiomas: growth pattern and residual lesions. Plast Reconstr Surg. 2011;127:1643-1648.

23. Denise W Metry, Management of infantile hemangiomas, Upto Date. 2013.

24. Darrow DH, Greene AK, Mancini AJ, et al. Diagnosis and Management of Infantile Hemangioma. Pediatrics. 2015;136:e1060-1065.

25. Püttgen K, Lucky A, Adams D, et al. Topical Timolol Maleate Treatment of Infantile Hemangiomas. Pediatrics. 2016;138-141.

26. Boon LM, MacDonald DM, Mulliken JB. Complications of systemic corticosteroid therapy for problematic hemangioma. Plast Reconstr Surg. 1999;104:1616-1623.

27. Breur JM, de Graaf M, Breugem CC, Pasmans SG. Hypoglycemia as a result of propranolol during treatment of infantile hemangioma: a case report. Pediatr Dermatol. 2011;28:169-171.

28. Tollefson MM, Frieden IJ. Early growth of infantile hemangiomas: what parents' photographs tell us. Pediatrics. 2012;130:e314-320.

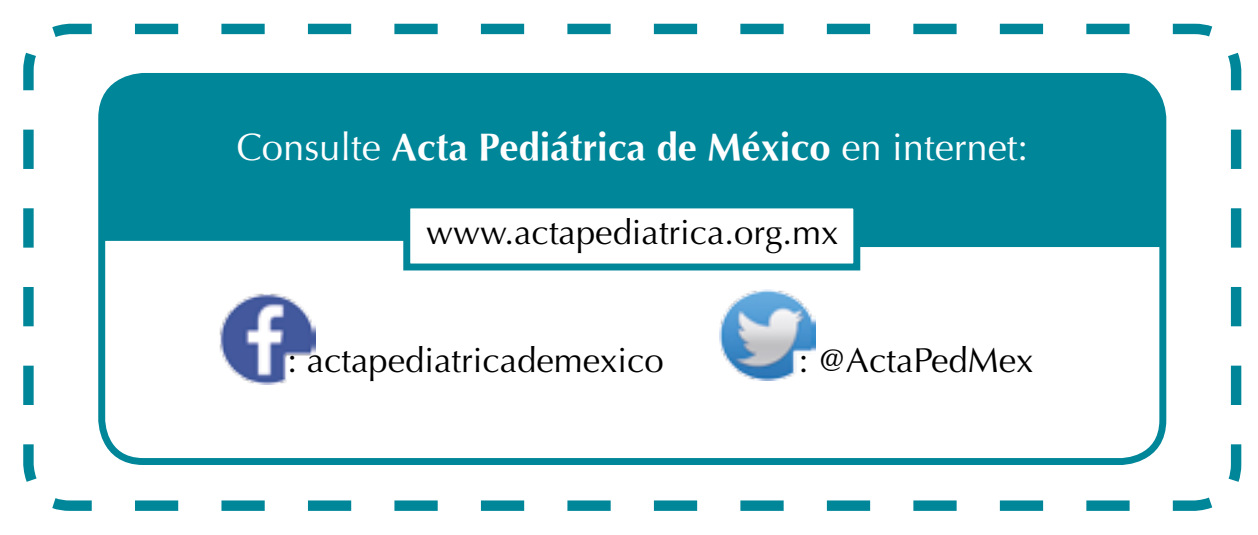

